\title{
Palmoplantar Lichen Planus A diagnosis to keep in mind
}

"Ricardo Ruiz-Villaverde, ${ }^{1}$ Beatriz Rueda-Villafranca, ${ }^{2}$ Marina Ruiz Galvez-Moreno ${ }^{1}$

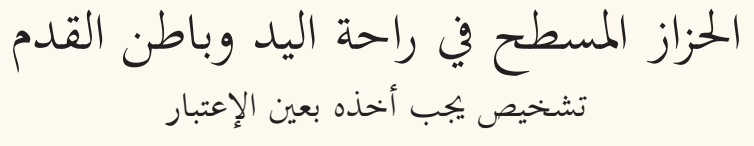

ريكاردو رويز-فيلافيردي، بياتريز رويدا فيلافرانكا، مارينا رويز غالفيز مورينو
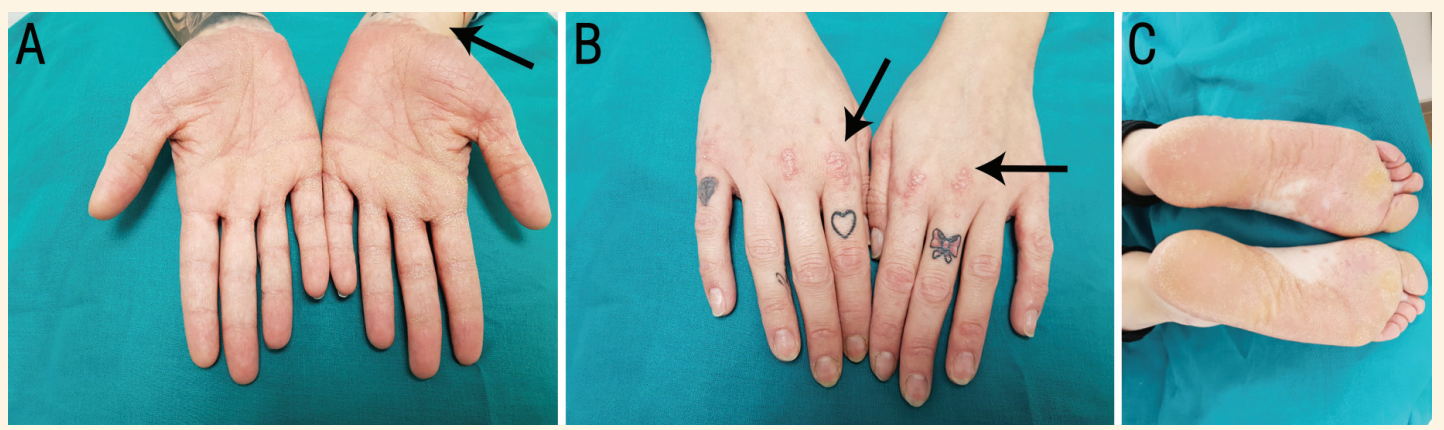

Figure 1: Photographs of the hands and feet of a 29-year-old female patient showing well-defined diffuse erythema (arrows) on both (A) palms and (B) dorsum sparing the fingertips and (C) well-defined erythematous patches sparing the plantar arch on both soles.

A 29-YEAR-OLD CAUCASIAN FEMALE PATIENT was referred to the Dermatology Outpatient Clinic of Hospital Universitario San Cecilio, Granada, Spain in 2018 with a pruriginous palmoplantar eruption over the previous five months that had not improved with the application of high-potency topical corticosteroids. She had no personal or family history of skin diseases. Recently, she denied the use of any medications.

Dermatological examination revealed the presence of a well-defined diffuse erythema on the palm of the hands that spared the fingertips and bilateral violaceous polygonal papules on the dorsum aspect of the hands [Figures 1A \& B]. On the soles of her feet, well-defined erythematous patches sparing the plantar arch were observed [Figure 1C]. No involvement of other skin areas or mucosa was noted. Laboratory tests including complete blood count, general biochemistry, venereal research laboratory tests, hepatitis $\mathrm{B}$ and $\mathrm{C}$ and HIV serology showed normal results. In addition, an allergic contact dermatitis patch test was negative.

Histolopathologic examination of a lesion on the dorsum of the right hand revealed orthokeratotic hyperkeratosis, irregular acanthosis, vacuolar degeneration of the basal layer and band-like lymphocytic infiltration on the papillary dermis [Figure 2].

Therefore, the patient was diagnosed with palmoplantar lichen planus (PPLP). Treatment with isotretinoin was proposed at a dose of $0.5 \mathrm{mg} / \mathrm{kg} /$ day with a substantial improvement three months later. At present, the patient remains asymptomatic with periodic consultations. Psoralen and ultraviolet A (PUVA) therapy for localised disease was refused by the patient for her inability to attend due to work-related reasons.

\section{Comment}

PPLP is an uncommon entity of low prevalence on the clinical spectrum of lichen planus with an incidence rate of $12.9-26 \% .{ }^{1}$ This disease has a male predilection and the average age of onset of 38 years with a range of 9-72 years. ${ }^{2}$

The absence of the typical polygonal violaceous papules, which were found on the dorsal aspect of both hands of the current patient, is not unusual; on the palms, the fingertips are usually spared. ${ }^{3,4}$ In general, of localised lichen planus cases become generalised with 

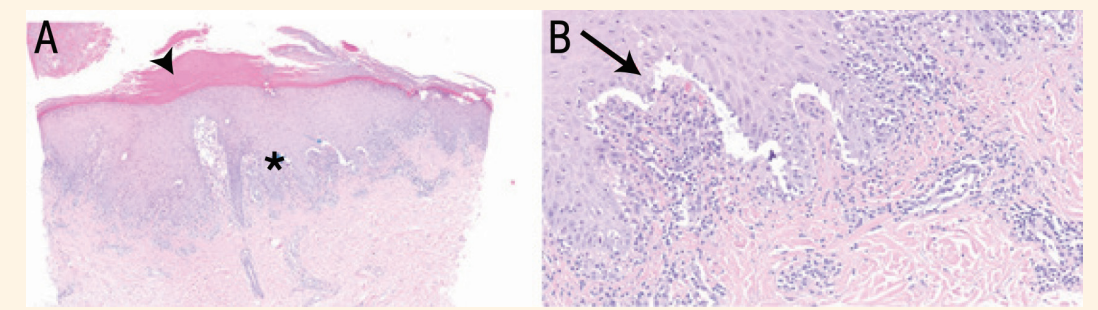

Figure 2: Haematoxylin and eosin stain at (A) x2 magnification showing orthokeratotic hyperkeratosis (arrowhead), irregular acanthosis and vacuolar degeneration of the basal layer (*) and at (B) x20 magnification showing the degeneration of the basal layer (arrow) and band-like lymphocytic infiltration on the papillary dermis.

the average time of evolution estimated to be between $1-4$ months. ${ }^{5}$

Five clinical types of PPLP have been described, namely eczematous, lichenoid, punctate keratotic, ulcerative and psoriasiform, which is the most frequent. ${ }^{1}$ There are isolated cases that have described the presence of vesicles, petechiae or umbilicated papules. ${ }^{4}$ Histolopathological studies are crucial to diagnose PPLP. The pathological characteristics of PPLP are shared with those of classic lichen planus. The thickness of the stratum lucidum of palms and soles has its clinical meaning in the absence of the characteristic Wickham's striae. ${ }^{5}$ Differential diagnosis should include psoriasis, hyperkeratotic eczema, diffuse keratoderma, keratosis punctata, lichen nitidus, granuloma annulare and secondary syphilis among others. ${ }^{4}$

A variable response to treatments, including retinoids, PUVA therapy, corticosteroids, methotrexate, cyclosporine and enoxaparin sodium, has been reported. ${ }^{4-6}$ It is necessary to establish a therapeutic protocol that assesses the risks and benefits of each patient.

\section{References}

1. Sinha S, Sarkar R, Garg VK. Palmoplantar lesions of lichen planus. Indian J Dermatol 2018; 63:57-61. https://doi.org/10.4103/ijd. IJD_161_17.

2. Gutte R, Khopkar U. Predominant palmoplantar lichen planus: A diagnostic challenge. Indian J Dermatol 2014; 59:343-7. https://doi.org/10.4103/0019-5154.135477.

3. Burillo-Martínez S, Tous-Romero F, Rodríguez-Peralto JL, Postigo-Llorente C. Palmoplantar lichen planus: The spectrum of clinical manifestations in a single patient. Actas Dermosifiliogr 2017; 108:790-2. https://doi.org/10.1016/j.ad.2017.01.019.

4. Solak B, Kara RO, Kosem M. Palmoplantar lichen planus successfully treated with acitretin. BMJ Case Rep 2015; 2015:bcr2015211115. https://doi.org/10.1136/bcr-2015-211115.

5. Kim MJ, Choi M, Na SY, Lee JH, Cho S. Two cases of palmoplantar lichen planus with various clinical features. J Dermatol 2010; 37:985-9. https://doi.org/10.1111/j.1346-8138.2010.00936.x.

6. Rieder E, Hale CS, Meehan SA, Leger M. Palmoplantar lichen planus. Dermatol Online J 2014; 20:13030/qt1vn9s55z. 